\title{
NEW ACCURACY LEVELS FOR SOLAR SYSTEM EPHEMERIDES
}

\author{
E. M. STANDISH AND X X NEWHALL \\ $J P L /$ Caltech \\ 301-150; Pasadena, CA 91109; USA
}

\begin{abstract}
DE403/LE403 is the latest JPL Planetary and Lunar Ephemeris. It represents a number of changes and improvements to previous JPL ephemerides: the reference frame is now that of the IERS, newer and more accurate observations are used in the adjustment process, some of the data reduction techniques have been refined, and improved dynamical modeling has been incorporated into the equations of motion. As a result, the internal accuracy of the inner four planets has been improved. Further, various measurements accurately tie Jupiter onto the IERS Reference Frame. In the future, use of CCD measurements and the Hipparcos Catalogue should improve the ephemerides of the outermost four planets.

DE403/LE403 has been integrated over 6000 years, from $3000 \mathrm{BC}$ to $3000 \mathrm{AD}$. A more condensed representation has been made from this, named DE404/LE404. It replaces DE102 as the new JPL "Long Ephemeris".
\end{abstract}

\section{Introduction}

The latest JPL Planetary and Lunar Ephemeris, DE403/LE403, was created in May 1995 and described by Standish et al. (1995). The JPL ephemerides are now numbered in the 400's, signifying that they are based upon the reference frame of the IERS Radio Catalogue. This has recently been made possible by including two new sets of data into the ephemeris adjustments: 1) $\triangle$ VLBI observations of the Magellan Spacecraft orbiting Venus and of the Phobos Spacecraft during its approach to Mars and 2) a frame-tie determination between previous JPL ephemerides and IERS Radio Catalogue. The internal accuracy of the ephemerides has also been improved. Recent radar-ranging observations to Mercury, Venus, and Mars 
have been acquired; the complete set of Viking Lander ranges is now used; VLA thermal emission measurements of the Jovian planets and $\triangle$ VLBI measurements of the Magellan, Phobos, and Ulysses spacecraft have been added to the data set; the tracking files of the Voyager 1 encounter of Jupiter have been re-reduced; the set of transit observations has been extended, including photoelectric transit measurements of Pluto; and Lunar Laser Ranging observations up to the present are included.

The data reduction processes and the dynamical modeling of the forces have also been refined: the surfaces of Mercury and Venus are modeled more accurately, the perturbations of 300 asteroids are now accounted for, and the formulation for integrating the lunar librations has been improved.

The IERS Reference Frame is discussed in Section II; the data sets to which the ephemerides are fit, in Section III; the improvements to the data reductions and to the dynamical modeling are discussed in Section IV; DE403 is compared with DE200 and DE102 in Section V; and the creation of DE404 is described in Section VI.

\section{The IERS Reference Frame}

The choice of the IERS Reference Frame for the ephemerides is advantageous for the following reasons:

- the ephemerides are now adjusted to a number of accurate $( \pm 0 ! 001$ to \pm 0 !005) observations which are referenced to the IERS Frame,

- the frame-tie between the JPL ephemerides and the IERS Frame has been accurately ( \pm 0 !003) measured,

- the IERS Frame itself is accurate: the source positions are now determined at the sub-milliarcsecond level,

- the IERS Frame is accessible and well-defined: the catalogue of source positions is published on an annual basis and is available worldwide,

- the IERS Frame is stable: an IAU directive to the IERS states that any further adjustments to the coordinates of the catalogue must be done in such a fashion that there is no net rotation introduced into the system as a whole, and

- the timing and polar motion information used for the orientation of the earth is referred to the IERS Frame.

The observations mentioned in the first two items above are discussed in the next section. They allow the ephemerides to be oriented automatically onto the IERS Frame. Of course, for the sake of consistency, any observations referred to any other external reference frame (e.g., optical FK5 Frame) must be appropriately adjusted. 


\section{The Observational Data}

The observational data to which the ephemerides have been fit are discussed in more detail by Standish et al. (1995). The sets include

- Optical observations: The transit observations are visual, photoelectric, and now, CCD. There are also astrometric plate observations of Pluto, astrolabe observations of Mars through Uranus, timings of occultations of stars by the rings of Uranus and the disk of Neptune.

- Radar ranging: The ranges to the terrestrial planets have been extended through 1991 for Mercury, 1990 for Venus, and 1993 for Mars. The modeling of these is discussed briefly in the next section.

- Spacecraft ranging: The ranging to Mars (Mariner 9 and Viking), Jupiter (Voyagers 1 and 2), and Mercury and Venus (Mariner 10) have been previously discussed by Standish et al. (1976, 1990, and 1995).

- Thermo-electric emission: These measurements of the Galilean satellites, Titan, Uranus and Neptune, taken at the VLA, are discussed by Muhleman et al. (1985) and by Berge et al. (1988). They refer the center of the thermally-emitting disk to the IERS reference radio sources with an accuracy of about $( \pm 0 ! 03)$.

- Spacecraft tracking files. These are the standard doppler and range data which are used by the JPL Navigation Team for determining spacecraft trajectories. If the spacecraft is close enough to the planet to be influenced by its gravity, the ephemeris of the target planet is adjusted as part of the orbit determination process.

- Laser-ranging to the moon. The twenty-five years of data show a general increase in accuracy from $30 \mathrm{~cm}$ in the early years to less than 3 $\mathrm{cm}$ at present.

- IERS-based $\triangle$ VLBI of Phobos approaching Mars and Magellan orbiting Venus: These observations have been reported by Folkner et al. $(994 a, b)$. In conjunction with the planet-centered spacecraft ephemeris, these observations provide a position of the planet wrt the IERS Frame which is generally accurate to about $( \pm 0 ! 003)$.

- Frame-tie measurement. (Folkner et al.., 1993a) have measured the frame-tie between JPL ephemerides and the IERS Reference Frame: the IERS Catalogue is connected to the VLBI antennae through the VLBI measurements; the antennae are connected to the LLR telescopes via geodetic ground surveys; the LLR telescopes are connected to the Moon by the LLR observations. Since the LLR data is sensitive to the positions of the sun, the whole inner planet system is connected to the Moon. Thus, the planetary positions are effectively linked to the IERS Catalogue. 


\section{Data Reduction and Dynamical Modeling}

Most of the standard data reduction and dynamical modeling in the JPL ephemeris improvement process have been discussed previously (see Standish, 1990 for the former; Newhall et al., 1983 for the latter). There have been some more recent refinements, however.

\subsection{OPTICAL DATA}

The observations are initially transformed onto the FK5 system. They are then corrected for more recent improvements to the value of precession. Finally, they are transformed onto the IERS Frame using a rotation matrix determined in the ephemeris solution itself.

\subsection{RADAR RANGES}

The radar-ranging to the surfaces of the terrestrial planets is directly affected by uncertainties in the topographical surface of the planet. Refinements come from improved modeling of these surfaces. For Mercury, an elliptically shaped equator has been fit and removed from the residuals, using the form, $-c \Delta \tau=R_{\text {Mercury }}+a \cos (2 \lambda)+b \sin (2 \lambda)$, where $\lambda$ is the longitude of the echo point on the surface of Mercury. For Venus, a topographical model of the surface, derived from Pioneer-Venus Orbiter data (Pettengill et al., 1980), was used.

\subsection{ASTEROID PERTURBATIONS}

There are quite a few asteroids whose perturbations produce a non-negligible effect upon the orbits of Mars and the Earth. For the JPL ephemerides, these are handled in two different ways.

The"Big 3": Ceres, Pallas, and Vesta, are handled individually: they are assumed to follow periodic Keplerian orbits: osculating elements are first transformed into mean elements (Williams, 1989); from the mean elements, positions for each asteroid are computed throughout one of its periods; these positions are fit with Chebychev polynomials, to be interpolated later during the integration; the interpolated positions are combined with a pre-assigned mass to compute the force which is applied to every planet. Corrections to the mass of each of the three asteroids may be solved for.

The 297 next most important asteroids are handled in a different manner: these asteroids are grouped according to approximate taxonomic class: $\mathrm{C}, \mathrm{S}$, or M; osculating elements are transformed into mean elements; the volume of each asteroid is computed from its estimated diameter, and a density is assigned according to its taxonomic class; vectors of the forces upon 
Mars, the earth and the moon are computed for each asteroid, summed over all of the asteroids in the class, and stored in a file to be interpolated during the integration of the equations of motion. Corrections to the density of each of the three classes may be solved for.

It should be noted in both cases, that the asteroids' contributions to the location of the center of mass of the solar system are accounted for.

The masses of the asteroids, Ceres, Pallas and Vesta, determined for DE403 are $4.64 \times 10^{-10}, 1.05 \times 10^{-10}$, and $1.34 \times 10^{-10}$ solar masses, respectively. These are $7 \%, 25 \%$ and $11 \%$ lower than those estimated by Standish and Hellings (1989). Interestingly, these newer results for Pallas and Vesta are quite close to the original values obtained by Schubart (1975); however, the uncertainties involved, approximately the sizes of the changes themselves, show this to be probably coincidental. On the other hand, the new value for the mass of Ceres agrees quite closely with results reported at this conference by Carpino and Knežević (1995) and by Viateau (1995).

For the densities of the three asteroid classes, the determinations for the M-class seem quite untrustworthy; consequently, that value was adopted as $5.0 \mathrm{gm} / \mathrm{cm}^{3}$ and not solved-for. The determined values for the C- and Sclasses, 1.80 and 2.40 , must be regarded as quite tentative.

\subsection{LUNAR MODELING}

The set of solution parameters includes orbits of moon and earth, physical librations, observatory and reflector coordinates, lunar gravity harmonics and moment of inertia combinations, lunar Love numbers and rotational dissipation, GM of earth+moon, precession and nutation of the earth, earth rotation corrections (mainly to the earlier data span) and drifts, and tidal acceleration parameters. Plate motion is imposed upon the stations.

Since the IERS celestial coordinates are not aligned with the J2000 equator it is necessary to solve for the offset of the equator from the zerodeclination plane of the IERS system (or equivalently, to solve for the offset of the rotation pole from the IERS north pole). The precession and obliquity rates have been adopted, the ecliptic plane is constrained, and the offsets are solved for. The offsets were treated as constant corrections to the two nutation components, which is an approximation. With $\mathrm{T}$ in centuries from J2000,

$\Delta \epsilon=-0 ! 00399-0 ! 024 T+$ periodic nutation corrections, and

$\sin \epsilon \Delta y=-0 ! 01536-0 ! 1193 T+$ periodic nutation corrections.

The periodic nutation corrections are adopted improvements in the nutations plus a solution for $18.6 \mathrm{yr}$ terms using the constraints of Williams et al. (1991). The LLR data also determine the difference between the orbital longitudes of the geocentric moon and sun. 
Tidal acceleration of the moon comes mainly from the ocean tides, but roughly $1 \%$ comes from dissipation in the moon. The present model for the orbit integration computes separate contributions from diurnal and semidiurnal earth tides. There are diurnal and semidiurnal tidal parameters (time delays) determined in the ephemeris adjustments. The tidal acceleration is computed to be $-25 ! 8 / \mathrm{cty}^{2}$ for the sum of the one lunar and two earth contributions.

\section{Comparisons with Previous Ephemerides}

\subsection{DE403 VS. DE200}

Comparisons between two ephemerides are usually best accomplished after the two frames have been aligned at some epoch; this avoids the masking of subtler differences by an overall global rotation. However, as determined by Folkner et al. (1993a), the frame-tie between DE403 and DE200 involves relatively small $\mathrm{x}-, \mathrm{y}$ - and $\mathrm{z}$-axis rotations:

$$
[+0 ! 002 \pm 0 ! 002,+0 ! 012 \pm 0 ! 003,+0 ! 006 \pm 0 ! 003] \text {. }
$$

This near alignment is probably from the influence of DE200 in establishing the original version of the radio source catalogue. As such, one may compare DE403 and DE200 without the need for any prior global alignment.

Standish et al. (1995) show plots of a comparison between DE403 and DE200, covering the valid time-span of DE200, 1750-2169. For the four innermost planets, the longitudes agree within \pm 0.005 at the present epoch, but there is a mean motion difference of about $+0 ! 05 /$ cty for the longitude difference, $\lambda_{403}-\lambda_{200}$; the latitudes agree within \pm 0 !02 or so throughout; the differences in geocentric distance are less than a kilometer during the past few decades and grow to tens of kilometers, a century or two away from the present.

For the outer planets, the agreement is progressively worse with each planet's distance from the sun. Over much of the present century, it is no more than $0 ! 3$; however, by J2000 it has grown to $0 ! 3$ for Neptune and to 3 " for Pluto. By the year 1750, the differences reach 1", 5", 7", 70", for Jupiter through Pluto, respectively.

For the moon, the difference of $-0 ! 9 / c t y^{2}$ dominates away from 1975 , the weighted mean epoch of the LLR data fit by DE200. In 1975, the longitude difference is only -0 !"002. A bias of about -4 meters is evident in the geocentric distance.

\subsection{DE403 VS. DE102}

The comparison between DE403 and DE102 does require an a priori global alignment since DE102 is 1950-based and is aligned only approximately onto the mean equator and dynamical equinox. Thus, the following discus- 
sion refers to DE102 after having been transformed to IAU J2000 System using the equinox offset of Fricke (1982) and the standard precession matrix (Lieske, 1979) and after having had the additional following rotation applied: [-0!0027, $-0 ! 1373,+0 ! 1412]$.

For the comparison, DE102 - DE404, the inner planets show a general mean motion difference of about $-0 ! 03 /$ cty in heliocentric longitude, while the latitudes agree to better than $-0 ! 1$ throughout; over the present several centuries, the heliocentric distances remain below $10 \mathrm{~km}$, but those for Mercury and Mars grow to over $100 \mathrm{~km}$ at times 2000 years away from the present. The moon is dominated by the difference of about $-0 ! 3 / c t y^{2}$ implying a difference of $-300 "$ in the year -1410 . The longitude differences for the five outer planets grow backward in time to about $-10 ",-20$ ", $+40 ",+300 "$ ", and $+400 "$ ", respectively, by the year -1000 ; the corresponding latitudes become $0 ! 25,0 ! 5,1 ", 10^{\prime \prime}$, and $100^{\prime \prime}$; the distances grow to \pm 2000 , $\pm 5000, \pm 40000,+10^{5}$ and $\pm 2 \times 10^{6}$.

\section{DE404/LE404, the New "JPL Long Ephemeris"}

DE404/LE404 is a condensed version of DE403/LE403; they both represent the identical ephemeris integration. However, DE404/LE404 is more condensed - in three ways:

- Earth nutations are not present on the DE404 file, since they may be obtained exactly from a formula.

- Lunar librations are not present on the DE404 file; instead, a separate file containing the librations has been made.

- The accuracy of the interpolating polynomials has been lessened for DE404 (though interpolation on the 64-day mesh points remains exact). For DE404/LE404, the interpolating accuracy is no worse than 25 meters for all planets and no worse than 1 meter for the moon adequate for nearly any application except for the processing of the most accurate observations.

For the full expanse, $3000 \mathrm{BC}$ to $3000 \mathrm{AD}, \mathrm{DE} 404 / \mathrm{LE} 404$ requires only 200 megabytes; DE403/LE403 would require over 550 megabytes to cover the same interval. DE404/LE404 will soon be available on CDROM's.

\section{Acknowledgements}

The research in this paper was carried out by the Jet Propulsion Laboratory, California Institute of Technology, under a contract with the National Aeronautics and Space Administration. 


\section{References}

Berge, G. L. , Muhleman, D. O., and Linfield, R. P. : 1988, "Very Large Array Observations of Uranus at $2.0 \mathrm{~cm}$ ", Astron. J. 96, \#1, 388-395.

Folkner, W. M. , Kroger, P. M. , and Iijima, B. A. , and Hildebrand, C. E. : 1992a, "VLBI measurement of Mars on 17 February 1989", JPL IOM 335.1-92-26.

Folkner, W. M. , Kroger, P. M. , and Iijima, B. A. , and Hildebrand, C. E. : 1992b, "VLBI measurement of Mars on 25 March 1989", JPL IOM 335.1-92-27.

Folkner, W. M. , Kroger, P. M. , and Hildebrand, C. E. : 1992c, "Preliminary results from VLBI measurement of Venus on September 12, 1990", , JPL IOM 335.1-92-25

Folkner, W. M. , Charlot, P. , Finger, M. H. , Williams, J. G. , Sovers, O. J. , Newhall, X $\mathrm{X}$, and Standish, E. M. : 1993a, "Determination of the extragalactic frame tie from joint analysis of radio interferometric and lunar laser ranging measurements", Astron. Astrophys. 287, 279-289.

Folkner, W. M. , Kroger, P. M. , and Iijima, B. A. : 1993b, "Results from VLBI measurement of Venus on March 29, 1992", JPL IOM 335.1-93-22

Folkner, W. M. , Kroger, P. M. , Iijima, B. A. , and Border, J. S. : 1994a, "Results from VLBI measurements of Venus on December 22 and December 23, 1991", JPL IOM 335.1-94-006

Folkner, W. M. , Border, J. S. , and Iijima, B. A. : 1994b, "Results from VLBI measurement of Venus on April 1, 1994", JPL IOM 335.1-94-014

Fricke, W. : 1982, "Determination of the Equinox and Equator of the FK5", Astron. Astrophys. 107, L13-L16

Carpino, M. and Knežević, Z.: 1995, this volume.

Lieske, J. H. : 1979, "Precession Matrix Based on IAU (1976) System of Astronomical Constants", Astron. Astrophys. 73, 282-284.

Muhleman, D. O. , Berge, G. L. , Rudy, D. J. , Niell, A. E. , Linfield, R. P. and Standish, E. M. : 1985, "Precise Position Measurements of Jupiter, Saturn and Uranus Systems with the Very Large Array", Cel. Mech. 37, 329-337.

Newhall, X X, Standish, E. M. and Williams, J. G. : 1983, "DE102: a numerically integrated ephemeris of the Moon and planets spanning forty-four centuries", Astron. Astrophys. 125, 150-167.

Pettengill, G. H. , Eliason, E. , Ford, P. G. , Loriot, G. B. , Masursky, H. , and McGill, G. E. : 1980, "Pioneer Venus Radar Results: Altimetry and Surface Properties", $J$. Geophys. Res. 85, A13, 8261-8270; table of values transmitted to the authors via W L Sjogren.

Schubart, J. : 1975, "The mass of Pallas", Astron. Astrophys. 39, 147-148.

Standish, E. M. , Keesey, M. S. W. and Newhall, X X : 1976, "JPL Development Ephemeris Number 96", JPL Tech. Rep. , 32-1603, Pasadena.

Standish, E. M. and Hellings, R. W. : 1989, "A Determination of the Masses of Ceres, Pallas and Vesta from their Perturbations upon the Orbit of Mars", Icarus 80, 326333.

Standish, E. M. : 1990, "The observational basis for JPL's DE200, the planetary ephemerides of the Astronomical Almanac", Astron. Astrophys. 233, 252-271.

Standish, E. M. , Newhall, X X, Williams, J. G. and Folkner, W. F. : 1995, "JPL Planetary and Lunar Ephemerides, DE403/LE403", JPL IOM 314.10-127.

Viateau, B. : 1995 , private communication.

Williams, J. G. : 1989, "Harmonic Analysis", BAAS 21, \# 3, 1009.

Williams, J. G. , Newhall, X X, and Dickey, J. O. ; 1991, "Luni-solar Precession: Determination from Lunar Laser Ranges", Astron. Astrophys. 241, L9-L12 